\title{
Diagnosis of multilayer structures and composite parts by multifrequency phase detection
}

\author{
Janusz Musiał ${ }^{1,}$, Serhiy Horiashchenko ${ }^{2}$, Kostyantin Horiashchenko ${ }^{2}$ and Joanna \\ W ilczarska ${ }^{1}$
}

${ }^{1}$ University of Science and Technology, Facoulty of M echanical Engineering, AI. prof. S. K aliskiego 7 85-789 Bydgoszcz, Poland

${ }^{2}$ K hmel nitsky N ational U niversity, Instytutska Street, 11, K hmel nytskyi, 29000, U kraine

\begin{abstract}
The article considers the issue of diagnosing the condition of multilayer elements and structures. Probing methods are considered. A multiphase detection method has been proposed to generate diagnostic information that allows the reflected signals from several consecutive layers to be obtained. The reflection distance is determined for each layer. The presentation of a two-dimensional model of structural sounding in the presence of two objects of study was considered. The three-dimensional model of search of objects of research of concrete designs considered in work provides probing by spherical waves of a detail, measurement of phase shifts of signals reflected from internal objects of research in three points. Division by means of one of mathematical models of signals are reflections from each object of research and determination of coordinates of objects of research and can by means of the mathematical model was represent. The equipment of a measuring instrument was developed to study the analytical multifrequency phase method of distance measurement. This system can be used to diagnose defects in multilayer structures that can transmit radio waves. A lso it can be used for quality control of the put coverings and for the analysis of thickness of composite materials, isolation layers in the equipment.
\end{abstract}

\section{Introduction}

A ging electrical systems are prevalent in today $₫$ society. A full scale evaluation of the problem is difficult. W iring was not normally considered in lifecycle maintenance. Over $90 \%$ of home fires are attributed to electrical fires, although it is not clear how many are due to installed wiring and how many to faulty plug-in consumer devices. In addition to the safety problem, aircraft wiring systems are a maintenance burden. For example known that between 1 million and 2 million man-hours are required at the operational level to troubleshoot and repair wiring system problems in the U.S. Navy alone each year. Highly trained technicians trouble shoot wiring problems using diagnostics methods that are many years old. V isual inspection, the most common traditional method, was determined to be insufficient to find fault. Especially hard to find faults in hidden wires. Time domain reflectometry (TDR), another traditional method for locating faults, was fond to be enough accurate but difficult to use $[1,2,3]$. High voltage test systems are able to locate even small faults, however they are very large and expensive. A lso, high voltage testing methods are able to reduce lifetime of wires or even create new faults $[1,2,3]$. That's why new methods are needed, and development funds have led to the emergence of a number of different techniques. In article described wire test methods that are suitable for handheld and compares their advantages and disadvantages. The methods compared are the time domain refleclomeler (TDR), frequency

* Corresponding author: Janusz.M usial@ utp.edu.pl 
domain reflectometer (FDR), mixed signal reflectometer (MSR), sequence time domain reflectometer (STDR), spread spectrum time domain reflectomeler (SSTDR).

\section{Problem statement}

Frequency domain reflectometry (FDR) sends a set of stepped-frequency sine waves down the wire. There are three types of frequency domain reflectometry that are commonly used in radar applications that are distinct in that they each measure a different sine wave properly (frequency, magnitude, phase) in order to determine distance. R elated methods are al so found in wire testing. These are Frequency M odulated Continuous W ave (FM CW) systems (which measure frequency shift), Phase Detection Frequency Domain Reflectometry (PD-FDR) systems (which measure phase shift), and Standing Wave Reflectometry (SWR) systems (which measure amplitude or nulls of the standing wave).

Considering the problem of probing the structure in general, it should be noted that finding the coordinates of the localization of the internal objects of the study should be carried out not by two coordinates, but by three. This is due to the fact that any solid body is a threedimensional figure. To establish the general relations, we formulate and solve first the twodimensional problem of probing the structure, and then we generalize the solution for the three-dimensional problem.

In the case of the two-dimensional task of probing the structure, the solid body will be a flat figure. To simplify, choose a rectangle with sides $L$ and $H$, shown in Fig.1.

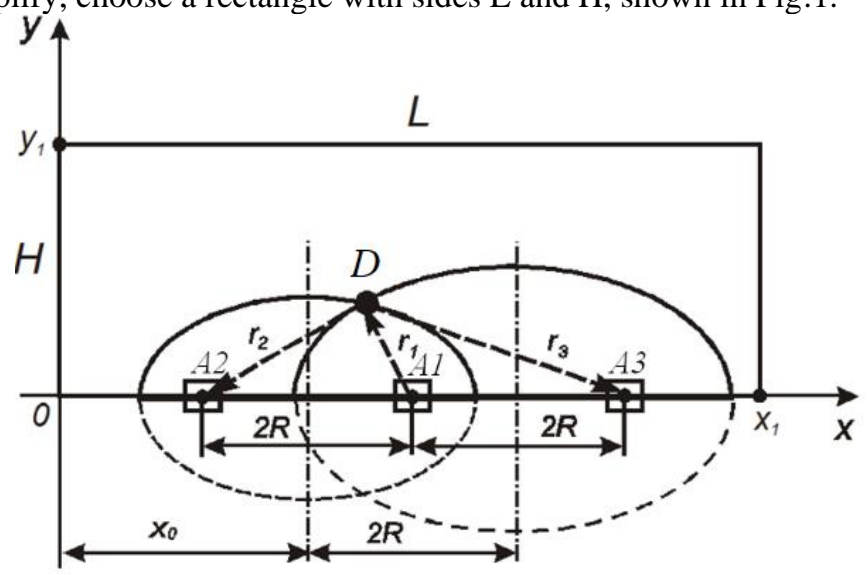

Fig.1. Representation of a two-dimensional problem of sounding a structure.

The probe signal is given by the radiating antenna $A_{l}$. to find the internal objects of the study. the harmonic test signal should be When using a phase-frequency approach. antenna $A_{2}$ is used to receive reflected signals. Both antennas must be fixed on one side of the rectangle at a distance of $2 R$ from each other. the harmonic signal of a given frequency on A 1 will form electromagnetic waves in a rectangular solid, which will propagate in the direction of the input angle of the antenna. In the absence of internal objects of study, the wave will be reflected from the sides of the rectangle, and the reflected waves will come to $A_{2}$, which will be added to each other at the connection point $A_{2}$. Thus, at the connection point $A_{2}$, a harmonic signal with a phase shift and amplitude characterizing some total signal $\bar{a}_{\Sigma_{3} 1}$ describing only a given geometric body will be received. If there is one object of study in the middle of this object, the reflection al so comes from it. In this case, another vector of the total signal $\bar{a}_{\Sigma \delta 1}$ will be obtained. If we subtract the vector $\bar{a}_{\Sigma \delta 1}$ from the vector $\bar{a}_{\Sigma_{3} 1}$, we get the vector of the signal $\bar{a}_{\Sigma 1}$ reflected only from the internal object of study: 


$$
\bar{a}_{\Sigma 1}=\bar{a}_{\Sigma \delta 1}-\bar{a}_{\Sigma_{31}}
$$

The phase shift of the received signal is proportional to the distance $r_{12}=r_{1}+r_{2}$ traveled by the probing signal from $A_{1}$ to the internal object $D$ (range $r_{1}$ ), and from $D$ to $A_{2}$ (range $r_{2}$ ). the geometric location of the nearby points corresponds to such a distance, which is an ellipse with foci at the locations of the antennas $A_{1}$ and $A_{2}$. That is, the obtained value of the distance corresponds to all points that are located on the half of the ellipse inside the flat figure. Thus, the measurement is not enough to find the coordinates of the location of the internal object of study

\section{Analysis of recent references and publications}

$V$ arious methods are used to analyze the state of the conductor line. The most well-known are the methods of reflectometry. Classic and easy to implement is pulse reflectometry or time dimension reflectometry (TDR). The development of digital technology has made it possible to introduce another type of reflectometry - phase reflectometry or frequency dimension reflectometry (FDR). A multi-frequency phase detection method based on damage reflections is a convenient tool for analyzing the condition of such a line. In modern research, the possibility of unambiguity in the interpretation of the information obtained by this method has been repeatedly pointed out [1-3]. In [1,2] it was shown that despite the fact that the reflection signals in the phase method at each frequency are the vector sum of harmonic signals at the sounding frequency, but the vector of the sum of these signals will vary depending on the signal frequency. The reason is to change the total phase shift angle in each case, for which it is determined by the increase of the phase shift angle for each reflection from the frequency. Therefore, as shown in $[2,7]$, there is a so-called rotation of the reflection vector (or equivalent in rate of change of the angle of displacement of the reflection phase) from the frequency with a certain angular velocity, which is determined by the expression in [8]:

Previous research has led to the development of phase methods designed to measure the distance of two, three or more objects. However, phase methods that measure the distances of three or more objects have significant drawbacks. Namely:

- to measure the distance it is necessary to scan a significant frequency range, which significantly limits the range of problems that can solve these methods;

- the accuracy of setting the minimum amplitude-frequency response of the total reflected signal (frequency response) is quite low due to its flat nature.

Therefore, this method has low accuracy, so it cannot meet modern requirements for measuring methods.

Therefore, it is necessary to find ways to reduce the frequency range of measurement signals and increase measurement accuracy. Improving the accuracy of measurement can be achieved by using computational measurement methods. A s a result of application of results of a small number of measurements, by calculations, values of necessary parameters come. This approach was used in the method of measuring the distance of two objects [1-3].

The research presented in the works is devoted to the development of mathematical models of the transformation of measuring signals, in order to determine the distance to each object of measurement and their characteristics. However, the mathematical model itself does not answer the question: how to make measurements; in what sequence to perform measuring operations; signal generation; measuring their parameters and conducting mathematical calculations. The answer to these questions is given by the method of measurement, namely the algorithm by which measurements and calculations are performed. The result of the development of the method should be the development of a device for phase sounding of the structure, designed for automated measurement of the parameters of the objects of study: their coordinates and reflection coefficients. $[5,6]$ Therefore, the task of further research is 
development of a phase method of sounding of a design and the structural scheme of the device of measurement of parameters of objects of research.

\section{Presenting main material}

To find the coordinates of the object of study $D$, it is proposed to use an additional signal receiver - antenna $A_{3}$, placing it on the other side of $A_{1}$ at a distance of $2 R$. When using $A_{3}$, the phase shift of the signal converted by it is proportional to the distance $r_{13}=r_{1}+r_{3}$ traveled by the probing signal from $B_{1}$ to the internal object of study $D$, (range $r_{l}$ ) and from $D$ to $A_{3}$, (range $r_{3}$ ). The ellipse equation can also be found for the obtained range, and it will differ from the ellipse equation for the $r_{I 2}$ range. At the intersection of both curves will be the internal object of study. By solving the system of equations for both ellipses, you can find the coordinates of the object of study.

Based on the above, we write a system of equations of ellipses for the two-dimensional problem of probing the structure

$$
\left\{\begin{array}{l}
\frac{4\left(x-x_{0}\right)^{2}}{\left(r_{1}+r_{2}\right)^{2}}+\frac{4 y^{2}}{\left(r_{1}+r_{2}\right)^{2}-4 R^{2}}=1 \\
\frac{4\left(x-x_{0}-2 R\right)^{2}}{\left(r_{1}+r_{3}\right)^{2}}+\frac{4 y^{2}}{\left(r_{1}+r_{3}\right)^{2}-4 R^{2}}=1 .
\end{array}\right.
$$

The system of equations will look like given that $r_{12}=r_{1}+r_{2}, r_{13}=r_{1}+r_{3}$,

$$
\left\{\begin{array}{l}
\frac{4\left(x-x_{0}\right)^{2}}{r_{12}^{2}}+\frac{4 y^{2}}{r_{12}^{2}-4 R^{2}}=1 \\
\frac{4\left(x-x_{0}-2 R\right)^{2}}{r_{13}^{2}}+\frac{4 y^{2}}{r_{13}^{2}-4 R^{2}}=1 .
\end{array}\right.
$$

When solving this system of equations, we choose the values of the roots that have a physical meaning and take into account some restrictions arising from Fig.1: the coordinates of the object of study may be within $x_{1} \geq x \geq 0 ; \quad y_{1} \geq y \geq 0 ， x_{0} \geq R ; x_{1} \geq x_{0}+3 R$; .

Based on this, we obtain the equations that determine the coordinates of the object of study $D$ :

$$
\begin{gathered}
x=\frac{\left(4 x_{0} R r_{12}+4 x_{0} R r_{13}-r_{12} r_{13}{ }^{2}+8 r_{12} R^{2}+r_{13} r_{12}{ }^{2}\right) R}{4\left(r_{12}+r_{13}\right)}, \\
y=\frac{\left.\left(r_{12}+r_{13}\right) \sqrt{\left(4 R^{2}-r_{13}\right)}{ }^{2}\right)\left(64 R^{4}+8 r_{12} R^{2} r_{13}-4 R^{2} r_{13}{ }^{2}-20 r_{12}{ }^{2} R^{2}-2 r_{13} r_{12}{ }^{3}+r_{13}{ }^{2} r_{12}{ }^{2}+r_{12}{ }^{4}\right)}{4 R},
\end{gathered}
$$

The situation is more complicated if there are two or more objects of research (Fig. 2). In this case, $A_{2}$ and $A_{3}$ will receive signals that are the sum of the signals reflected from each object of study. Thus, there is a problem of separating the signals reflected from each object of measurement.

This problem can be solved by one of the methods presented in the works. A fter dividing each signal, the distances are determined $r_{12}=r_{1}+r_{2}, r_{13}=r_{1}+r_{3}, r_{12}^{\prime}=r_{1}^{\prime}+r_{2}^{\prime}$, $r_{13}^{\prime}=r_{1}^{\prime}+r_{3}^{\prime}$ etc. 


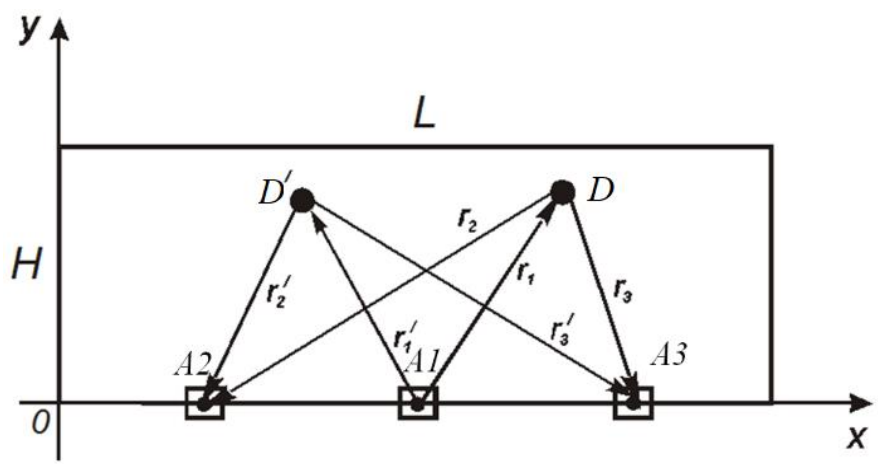

Fig.2. Representation of a two-dimensional model of structural sounding in the presence of two objects of research.

Then the task is reduced to recording the systems of equations (3) for each obtained pair of distances and finding the coordinates of the internal objects of the study.

When moving to the three-dimensional problem of probing the structure, it is necessary to use one radiating antenna to emit the probing signal $A_{1}$ and three antennas $A_{2}, A_{3}, A_{4}$ to receive the reflected signals, and they must be located on more than one line. It is proposed to place the emitter and two receivers on one line, and the third receiver on a line perpendicular to the line connecting the first two receivers, and passes through the point of installation of the emitter $[10,11]$. All distances between the emitter and receivers are the same and equal to $2 R$ (Fig.3).

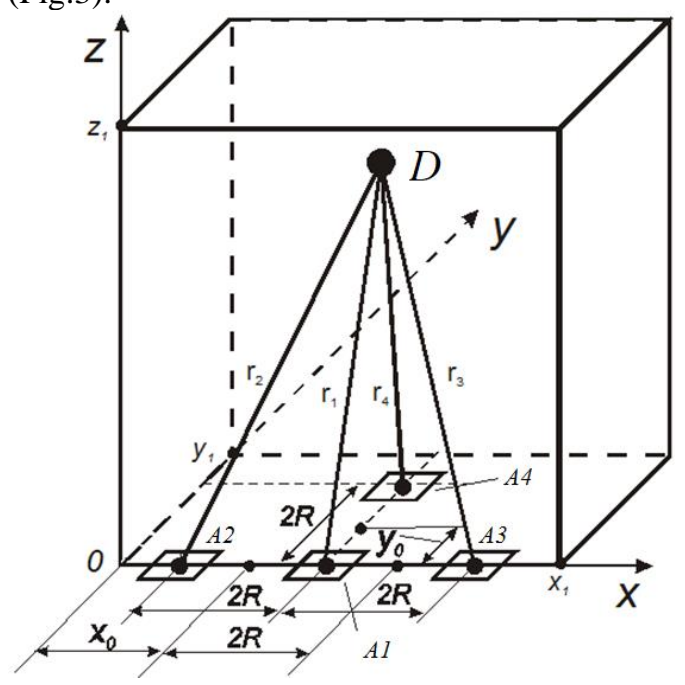

Fig.3. Representation of a three-dimensional problem of probing the structure.

The coordinates of the object of study in the three-dimensional problem will be determined by the point of intersection of three ellipsoids of rotation, the axes of rotation of two of which lie on the $X$ axis, and the axis of rotation of the third is perpendicular to the $X$ axis and passes through the radiating antenna $A_{1}$. [10, 11].

In this case, the system of ellipsoid equations for the three-dimensional problem will be as follows (6): 


$$
\left\{\begin{array}{l}
\frac{4\left(x-x_{0}\right)^{2}}{\left(r_{1}+r_{2}\right)^{2}}+\frac{4 y^{2}}{\left(r_{1}+r_{2}\right)^{2}-4 R^{2}}+\frac{4 z^{2}}{\left(r_{1}+r_{2}\right)^{2}-4 R^{2}}=1 \\
\frac{4\left(x-x_{0}-2 R\right)^{2}}{\left(r_{1}+r_{3}\right)^{2}}+\frac{4 y^{2}}{\left(r_{1}+r_{3}\right)^{2}-4 R^{2}}+\frac{4 z^{2}}{\left(r_{1}+r_{3}\right)^{2}-4 R^{2}}=1 \\
\frac{4\left(x-x_{0}-R\right)^{2}}{\left(r_{1}+r_{4}\right)^{2}-4 R^{2}}+\frac{4\left(y-y_{0}\right)^{2}}{\left(r_{1}+r_{4}\right)^{2}}+\frac{4 z^{2}}{\left(r_{1}+r_{4}\right)^{2}-4 R^{2}}=1 .
\end{array}\right.
$$

If we accept that $r_{12}=r_{1}+r_{2}, r_{13}=r_{1}+r_{3}, r_{14}=r_{1}+r_{4}$, then we get a system of equations (7):

$$
\left\{\begin{array}{l}
\frac{4\left(x-x_{0}\right)^{2}}{r_{12}^{2}}+\frac{4 y^{2}}{r_{12}^{2}-4 R^{2}}+\frac{4 z^{2}}{r_{12}^{2}-4 R^{2}}=1 \\
\frac{4\left(x-x_{0}-2 R\right)^{2}}{r_{13}^{2}}+\frac{4 y^{2}}{r_{13}^{2}-4 R^{2}}+\frac{4 z^{2}}{r_{13}^{2}-4 R^{2}}=1 \\
\frac{4\left(x-x_{0}-R\right)^{2}}{r_{14}^{2}-4 R^{2}}+\frac{4\left(y-y_{0}\right)^{2}}{r_{14}^{2}}+\frac{4 z^{2}}{r_{14}^{2}-4 R^{2}}=1
\end{array}\right.
$$

Given the constraints: $x_{1} \geq x \geq 0, \quad y_{1} \geq y \geq 0, \quad z_{1} \geq z \geq 0$, $x_{0} \geq R, x_{1} \geq x_{0}+3 R, y_{1} \geq y_{0}+R$, the solution of the system of equations (7) gives the following roots for determining the coordinates of the object of study in a three-dimensional system:

$$
\begin{gathered}
x=\frac{R\left(4 x_{0} R r_{12}+4 x_{0} R r_{13}+8 r_{12} R^{2}+r_{13} r_{12}^{2}-r_{12} r_{13}^{2}\right)}{4\left(r_{12}+r_{13}\right)} \\
y=\frac{r_{12}+r_{13}}{4 R^{2}}\left(4 r_{12} R^{2} y_{0}-r_{12} r_{14}^{2} y_{0}+4 y_{0} R^{2} r_{13}-y_{0} r_{14}^{2} r_{13}+r_{14} \sqrt{\Omega}\right) ; \\
z=\frac{r_{12}+r_{13}}{4 R^{2} \sqrt{S}} .
\end{gathered}
$$

where:$$
\Omega=64 R^{6}-4 y_{0}^{2} R^{2} r_{13}^{2}+2 y_{0}^{2} r_{14}^{2} r_{12} r_{13}-8 y_{0}^{2} R^{2} r_{12} r_{13}+y_{0}^{2} r_{14}^{2} r_{12}^{2}+y_{0}^{2} r_{14}^{2} r_{13}^{2}-
$$$$
-12 r_{12}^{2} R^{4}-4 y_{0}^{2} R^{2} r_{12}^{2}+r_{12}^{4} R^{2}-12 R^{4} r_{13}^{2}-r_{14}^{2} R^{2} r_{13}^{2}+R^{2} r_{13}^{4}-
$$$$
-2 r_{14}^{2} R^{2} r_{12} r_{13}+2 r_{12}^{2} R^{2} r_{13}^{2}-r_{14}^{2} R^{2} r_{12}^{2}+8 R^{4} r_{12} r_{13} \text {; }
$$$$
S=\left(-8 y_{0} R^{2} r_{13} r_{14}-8 r_{12} R^{2} y_{0} r_{14}+2 r_{12} r_{14}^{3} y_{0}+2 y_{0} r_{14}^{3} r_{13}\right) \sqrt{\Omega}-64 R^{6} r_{14}^{2}+12 y_{0}^{2} R^{2} r_{13}^{2} r_{14}^{2}-
$$$$
-4 r_{12} r_{14}^{4} y_{0}^{2} r_{13}-16 y_{0}^{2} R^{4} r_{13}^{2}+24 r_{12} R^{2} y_{0}^{2} r_{14}^{2} r_{13}-16 r_{12}^{2} R^{4} y_{0}^{2}-2 r_{12}^{2} r_{14}^{4} y_{0}^{2}-2 y_{0}^{2} r_{14}^{4} r_{13}^{2}+12 R^{4} r_{14}^{2} r_{12}^{2}+
$$$$
+12 r_{12}^{2} R^{2} y_{0}^{2} r_{14}^{2}+32 r_{12} R^{4} y_{0}^{2} r_{13}+4 r_{12}^{4} R^{4}-80 R^{6} r_{13}^{2}+4 R^{4} r_{13}^{4}-80 r_{12}^{2} R^{6}-R^{2} r_{14}^{2} r_{12}^{4}+12 R^{4} r_{14}^{2} r_{13}^{2}(12)
$$$$
+R^{2} r_{14}^{4} r_{13}^{2}-R^{2} r_{14}^{2} r_{13}^{4}+2 R^{2} r_{14}^{4} r_{12} r_{13}-2 R^{2} r_{14}^{2} r_{13}^{2} r_{12}^{2}+R^{2} r_{14}^{4} r_{12}^{2}-8 R^{4} r_{14}^{2} r_{12} r_{13}+24 r_{12}^{2} R^{4} r_{13}^{2}-
$$$$
-8 r_{12}^{3} R^{4} r_{13}-8 R^{4} r_{12} r_{13}^{3}+32 R^{6} r_{12} r_{13}-r_{12}^{2} r_{13}^{4} R^{2}+2 r_{12}^{3} r_{13}^{3} R^{2}-r_{13}^{2} r_{12}^{4} R^{2}+256 R^{8} \text {. }
$$

If there are two or more objects of study, it is necessary to apply the method described for a two-dimensional system, ie to determine the coordinates of each object of study independently of the others, using information about the phases of the signals reflected from the objects of study. 
To move to the phase relations in the system of equations (7) we substitute the values $r_{12}$, $r_{13}, r_{14}$ using the expression according to which $r_{12}=\frac{\varphi_{12}}{4 \pi} \lambda, r_{13}=\frac{\varphi_{13}}{4 \pi} \lambda, r_{14}=\frac{\varphi_{14}}{4 \pi} \lambda$.

We obtain a new system of equations that according with $[10,11]$.

$$
\left\{\begin{array}{l}
\frac{16 \pi^{2}\left(x-x_{0}\right)^{2}}{\varphi_{12}^{2} \lambda^{2}}+\frac{16 \pi^{2} y^{2}}{\varphi_{12}^{2} \lambda^{2}-16 \pi^{2} R^{2}}+\frac{16 \pi^{2} z^{2}}{\varphi_{12}^{2} \lambda^{2}-16 \pi^{2} R^{2}}=1 \\
\frac{16 \pi^{2}\left(x-x_{0}-2 R\right)^{2}}{\varphi_{13}^{2} \lambda^{2}}+\frac{16 \pi^{2} y^{2}}{\varphi_{13}^{2} \lambda^{2}-16 \pi^{2} R^{2}}+\frac{16 \pi^{2} z^{2}}{\varphi_{13}^{2} \lambda^{2}-16 \pi^{2} R^{2}}=1 \\
\frac{16 \pi^{2}\left(x-x_{0}-R\right)^{2}}{\varphi_{14}^{2} \lambda^{2}-16 \pi^{2} R^{2}}+\frac{16 \pi^{2}\left(y-y_{0}\right)^{2}}{\varphi_{14}^{2} \lambda^{2}}+\frac{16 \pi^{2} z^{2}}{\varphi_{14}^{2} \lambda^{2}-16 \pi^{2} R^{2}}=1
\end{array}\right.
$$

From here we determine the coordinates of the object of study

$$
\begin{gathered}
x=\frac{\lambda^{2} \varphi_{12} \varphi_{13}\left(\varphi_{12}-\varphi_{13}\right)}{16 \pi^{2} R\left(\varphi_{12}+\varphi_{13}\right)}+\frac{2 R^{2} \varphi_{12}}{\varphi_{12}+\varphi_{13}}+x_{0}{ }^{\prime} \\
y=-\frac{\varphi_{14} \sqrt{G}}{16 \pi^{2} R^{2}\left(\varphi_{12}+\varphi_{13}\right)}+\frac{y_{0} \lambda^{2} \varphi_{14}}{16 \pi^{2} R^{2}}-y_{0}{ }^{\prime} \\
z={\frac{\sqrt{F}}{16 \pi^{2} R^{2} \lambda\left(\varphi_{12}+\varphi_{13}\right)}}^{\prime}
\end{gathered}
$$

where:

$$
\begin{aligned}
G= & 1024 \pi^{2} R^{6}+R^{2} \lambda^{4}\left(\varphi_{12}^{2}+\varphi_{13}^{2}\right)^{2}+2 \lambda^{2}\left(y_{0}^{2}-R^{2}\right) \varphi_{12} \varphi_{13}\left(\lambda^{2} \varphi_{14}^{2}-16 \pi^{2} R^{2}\right)- \\
- & \left(16 \pi^{2} \lambda^{2} R^{2}\left(y_{0}^{2}-3 R^{2}\right)\left(\varphi_{12}^{2}+\varphi_{13}^{2}\right)+\lambda^{4}\left(y_{0}^{2}-R^{2}\right) \varphi_{14}^{2}\right), \\
& F=\left(3 \pi^{2} R^{2} y_{0} \lambda^{2}-2 y_{0} \lambda^{4} \varphi_{14}^{2}\right)\left(\varphi_{12}+\varphi_{13}\right) \varphi_{14} \sqrt{G}+ \\
& +16 \pi^{2} R^{4} \lambda^{4}\left(\varphi_{12}^{4}+\varphi_{13}^{4}\right)-R^{2} \lambda^{6} \varphi_{13}^{2}\left(\varphi_{12}^{4}-\varphi_{14}^{4}\right)+ \\
& +\left[48 \pi^{2} R^{2} \lambda^{4} \varphi_{14}^{2}\left(R^{2}+y_{0}^{2}\right)-\left(2 y_{0}^{2} \lambda^{6} \varphi_{14}^{4}-256 \pi^{4} R^{4} y_{0}^{2} \lambda^{2}\right)\right] \times \\
& \times\left(\varphi_{12}^{2}+\varphi_{13}^{2}\right)-R^{2} \lambda^{6} \varphi_{13}^{4}\left(\varphi_{12}^{2}+\varphi_{14}^{2}\right)-R^{2} \lambda^{6} \varphi_{12}^{2} \varphi_{14}^{2}\left(\varphi_{12}^{2}-\varphi_{14}^{2}\right)+ \\
& +2 R^{2} \lambda^{6} \varphi_{12}^{2} \varphi_{13}^{2}\left(\varphi_{12} \varphi_{13}-\varphi_{14}^{2}\right)-1280 \pi^{4} R^{6} \lambda^{2}\left(\varphi_{12}+\varphi_{13}\right)- \\
& -32 \pi^{2} R^{4} \lambda^{4} \varphi_{12}^{2} \varphi_{13}\left(\varphi_{12}-3 \varphi_{13}\right)+\left[2 \lambda^{6}\left(R^{2}-2 y_{0}^{2}\right) \varphi_{14}^{4}-\right. \\
& \left.-32 \pi^{2} R^{2} \lambda^{4}\left(R^{2}-3 y_{0}^{2}\right) \varphi_{14}^{2}+512 \pi^{4} R^{4} \lambda^{2}\left(R^{2}-y_{0}^{2}\right)\right] \times \\
& \times \varphi_{12} \varphi_{13}+1024 \pi^{4} R^{6} \lambda^{2} \varphi_{14}^{2}+16384 \pi^{6} R^{8}
\end{aligned}
$$

Therefore, to determine the parameters of an object in three-dimensional space, it is necessary to use an additional receiving antenna and solve a system of equations with respect to the three coordinates of space.

The three-dimensional model of search of objects of research of concrete designs considered in work provides probing by spherical waves of a detail, measurement of phase shifts of signals reflected from internal objects of research in three points. Division by means of one of mathematical models of signals are reflections from each object of research and determination of coordinates of objects of research and can by means of the mathematical model was represent. A ccordingly, the method of finding the coordinates and characteristics of the objects of study will be as follows.

The first stage of the method of phase probing of the structure will be the generation or synthesis of measuring signals. A s follows from the above, you need to synthesize signals in some frequency range. Depending on the number of subjects, the required measurement 
accuracy, the frequency range can be set higher or lower. To determine the frequency band, it is necessary to set the accuracy of the coordinate measurement $\Delta$, with which you can determine the accuracy of determining the phase shift and amplitude $\Delta_{\varphi^{\prime}} \Delta_{u}$ according to. According to the values $\Delta_{\varphi}, \Delta_{u}$, determine the step of the frequency grid $\Delta_{\omega}$ and frequency band $\Omega$. After these calculations, the synthesis of the measuring signal is performed. It is possible to use two types of signals. First, harmonic signals - the so-called discrete set of frequencies. Second, the signals from the rectangular envelope spectrum signals of the form of periodic functions $\sin (\omega t) / \omega t$ and $(1-\cos (\omega t)) / \omega t$. In the first case, the simplicity of the device circuit is achieved, in the second the speed of measurement.

The second stage is probing the object of probing the structure with measuring signals and receiving the reflected signals at three points. This step requires the location of the antennas at clearly defined distances to achieve the required measurement accuracy.

In the third stage, the phase shifts and amplitudes of the reflected signals are measured at three points. A phase meter and a voltmeter are used for measurement. To increase the speed, you can use three pairs of measuring units - measuring phase shift and voltage. To reduce the complexity of the equipment, you can use one unit for measuring phase shift and voltage and provide switching antenna connections.

The last stage will be to calculate the phase shifts and amplitudes of the signals reflected from each object of study and determine the coordinates of their location. [9]

\section{Results of experimental studies}

The problem of increasing the accuracy of measuring the distance to the boundaries of the separation of subsurface layers is proposed to be solved by taking into account their electrophysical properties. To do this, the subsurface medium is probed along the vertical axis with signals with linear-frequency modulation with stepwise frequency change in a narrow frequency range. The scheme of sounding is conducted in fig.4. The number of frequencies must be twice the number of boundaries of the subsurface layers. The phase shifts and amplitudes of the total signals reflected from all boundaries of the separation of subsurface layers are measured.

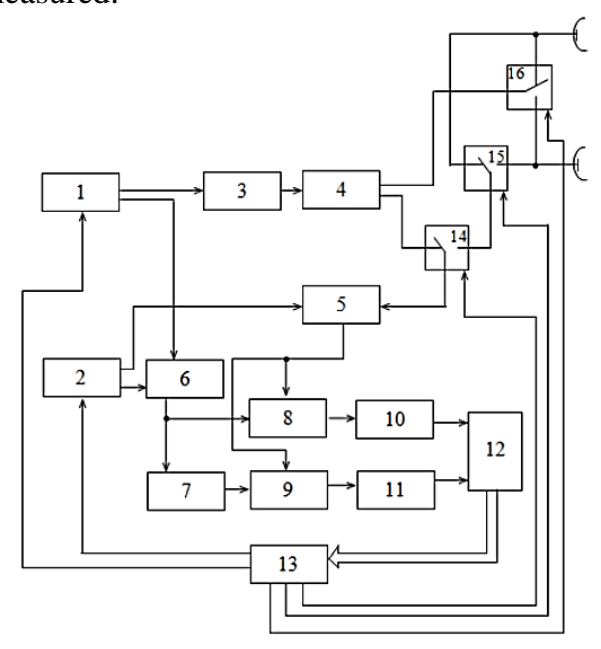

Fig.4. Subsurface radar sounding device: 1 - frequency-modulated signal generator, 2-reference signal generator, 3 - power amplifier, 4 - coupler, 5, 6 - first and second mixer, respectively, 7 - phase rotator, 8, 9 - first and second phase detectors, 10, 11 - the first and second low-pass filters, 12 - 
analog-to-digital converter, 13 - electronic computing device, 14, 15, 16 - the first, second, third highfrequency switches, 17, 18 - the first, the second receiving - transmitting antennas respectively.

The proposed technical solution provides the ability to anal yze the reflected signals taking into account their phase structure. Increase the reliability of determining the characteristics of subsurface objects due to the presence of a measuring channel and reference signal channel, two phases' detectors, phase shifter and two low-pass filters quadrature channels, as well as controlled from an electronic computing device by means of high-frequency switches of two transceiver antennas [11]. It can emit and receive signals in two mutually perpendicular directions of polarization allows to obtain a complete polarization matrix of radio waves scattering by its subsurface.

Using a mathematical model of the multifrequency phase method [12-14], the distances and reflection coefficients of all boundaries of the separation of subsurface layers are calculated by the formulas (19-20) with use $[14,15]$.

$$
\begin{gathered}
l_{1}=\frac{\varphi_{1} v}{4 \pi f}, l_{2}=\frac{\varphi_{2} v}{4 \pi f}, l_{3}=\frac{\varphi_{3} v}{4 \pi f}, \ldots, l_{s}=\frac{\varphi_{s} v}{4 \pi f} . \\
k_{1}=\frac{a_{1}}{a}, k_{2}=\frac{a_{2}}{a}, k_{3}=\frac{a_{3}}{a}, \ldots, k_{s}=\frac{a_{s}}{a} .
\end{gathered}
$$

In expressions (18-20) the speed of propagation of electromagnetic waves in different media is assumed to be the same. H owever, in different environments it is different. To clarify the measurement results, it is necessary to determine the real values of the velocities of electromagnetic waves in different subsurface environments. To do this, the following measurements and calculations are performed $l_{о \Pi}$. As a result of measuring the phase shift $\varphi_{v}$ between the probing harmonic signal at the frequency $f_{o \Pi}$ and the received signal we obtain, the value of the speed of propagation of the electromagnetic wave can be calculated in the first subsurface layer:

$$
v_{1}=\frac{2 \pi l_{o \Pi} f_{\text {оп }}}{\varphi_{v}}
$$

U sing the value of the speed of propagation of the radio wave in the first subsurface layer, the value of the real part of the dielectric constant is cal culated:

$$
\operatorname{Re} \varepsilon_{1}=\varepsilon_{1}^{\prime}=\frac{c}{v_{1}} \text {. }
$$

If the amplitude of the signal coming to the receiving antenna $A_{p r}$, is measured, and the amplitude of the signal emitted by the transmitting antenna $A_{v p}$, is known, then the value of the specific attenuation in the first subsurface layer is calculated by the expression:

$$
\alpha_{1}=\frac{l}{l_{o \Pi}} \ln \frac{A_{p r}}{A_{v p}}
$$

The calculation of the distance to the boundary of the separation of the first and second layers is carried out by expression:

$$
l_{1 \mathrm{y}}=\frac{\varphi_{1} v_{1}}{4 \pi f} .
$$

In this expression we substitute the value of the speed of propagation of the radio wave which is calculated according to the expression (22).

The value of the specific attenuation $\alpha_{1}$ and the calculated distance $l_{l y}$ is used for the value of the amplitude of the signal that came to the boundary of the separation of the first and second subsurface layers by expression, we find:

$$
A_{v p 21}=A_{v p} e^{-\alpha_{1} l_{o п}}
$$


The next step is to calculate the amplitude of the signal reflected from the separation boundary of the first and second subsurface layers using the value of the amplitude of the signal reflected from the first boundary $A_{1}$ that came to the receiving antenna, by expression:

$$
A_{v p 21}=\frac{A_{1}}{e^{-\alpha_{1} l_{\mathrm{on}}}} .
$$

The obtained values are used to calculate the reflection coefficient from the first boundary of the separation of the two media:

$$
K_{v p 21}=\frac{A_{p r 21}}{A_{v p 21}}
$$

Using the value of the reflection coefficient from the first boundary and the dielectric constant of the first layer, the real part of the dielectric constant of the second layer is calculated by the expression:

$$
\dot{\varepsilon_{2}}=\frac{\varepsilon_{1}\left(K_{v p 21}-1\right)^{2}}{\left(K_{v p 21}+1\right)^{2}}
$$

Using the value of the real part of the dielectric constant of the second layer, the speed of propagation of the second layer is calculated:

$$
v_{2}=\frac{c}{\dot{\varepsilon_{2}}}
$$

The calculation of the depth to the boundary of the separation of the second and third layers is carried out by the expression:

$$
l_{2}=l_{1}+\frac{\left(\varphi_{2}-\varphi_{1}\right) v_{2}}{4 \pi f} .
$$

The value of the coefficient of passage of the first boundary in the expression is calculated:

$$
K_{p \kappa 21}=1-K_{v p 21}
$$

The material of the second layer is determined by the value of the actual part of the dielectric constant, and the value of the conductivity of the layer is selected using tabular data $[6,9,10] \sigma_{2}$. The attenuation value in the second layer is calculated using the conductivity value:

By expression:

$$
\alpha_{2}=\frac{2 \pi f}{c} \cdot \sqrt{\frac{\varepsilon_{2}^{\prime}}{2}\left(\sqrt{1+\left(\frac{\sigma_{2}}{2 \pi f \varepsilon_{0} \varepsilon_{2}}\right)^{2}}-1\right)}
$$

$$
K_{v p 32}=\frac{a_{2}}{a} \cdot \frac{e^{2}\left(\alpha_{1} l_{1}+\alpha_{2}\left(l_{2}-l_{1}\right)\right)}{\left(K_{v p 21}-1\right)^{2}}
$$

The value of the reflection coefficient from the second boundary of the separation of subsurface layers is calculated.

Continuing similar considerations, to find the true part of the dielectric constant of the $S$-th layer uses the expression:

$$
\dot{\varepsilon_{S}}=\frac{\dot{\varepsilon_{S-1}}\left(K_{v p S, S-1}-1\right)^{2}}{\left(K_{v p S, S-1}+1\right)^{2}}
$$

where the reflection coefficient from the $S$-th boundary is by expression:

$$
K_{v p S, S-1}=\frac{a_{2}}{a} \cdot \frac{e^{2}\left(\alpha_{1} l_{1}+\alpha_{2}\left(l_{2}-l_{1}\right)+\cdots+\alpha_{S}\left(l_{S}-l_{S-1}\right)\right)}{\left(\left(1-K_{1}\right)\left(1-K_{1}\right) \ldots\left(1-K_{V P S-1, S-2}\right)\right)^{2}} .
$$


The speed of propagation of the radio $S$-th layer:

Range to the $S$-th limit:

$$
v_{S}=\frac{\mathrm{c}}{\varepsilon_{S}^{\prime}} \text {. }
$$

$$
l_{s}=l_{s-1}+\frac{\left(\varphi_{S}-\varphi_{s-1}\right) v_{s}}{4 \pi f} .
$$

Signals that are reflected from the previous boundaries of the separation of the two media in the reverse passage of the reflected signal can be ignored based on the following considerations $[16,17,18]$. As studies have shown in the previous section, the reflection coefficients differ from the transmission coefficients by approximately one order of magnitude and the re-reflected signals, given the attenuation of the signals, will not create any appreciable effect on the total signal.

However, if we consider the problem of probing the structure in general, it should be noted that finding the coordinates of the localization of subsurface objects should be carried out not in two coordinates [19], but in three coordinates, because any solid is a threedimensional figure [20]. To establish general relations, it is necessary to formulate and solve a two-dimensional problem of probing the structure, and then generalize the solution for the three-dimensional problem.

The equipment of a measuring instrument was developed to study the analytical multifrequency phase method of distance measurement and shown in fig. 5 .

Laboratory equipment works as follows. Controls the operation of equipment microcontroller control unit. It sets the frequency codes for the frequency synthesizer. The signal frequency varies from $10 \mathrm{kHz}$ in increments of $10 \mathrm{kHz}$ to $10 \mathrm{M} \mathrm{Hz}$. The controls change the value of the current frequency. The frequency and waveform are controlled by a digital oscilloscope. The transceiver unit coordinates the signal scheme. From the output of the receiver the signal is fed to the second input of the phase meter and voltmeter. To measure the phase shift, a reference signal from the frequency synthesizer is fed to the first input of the phase meter.
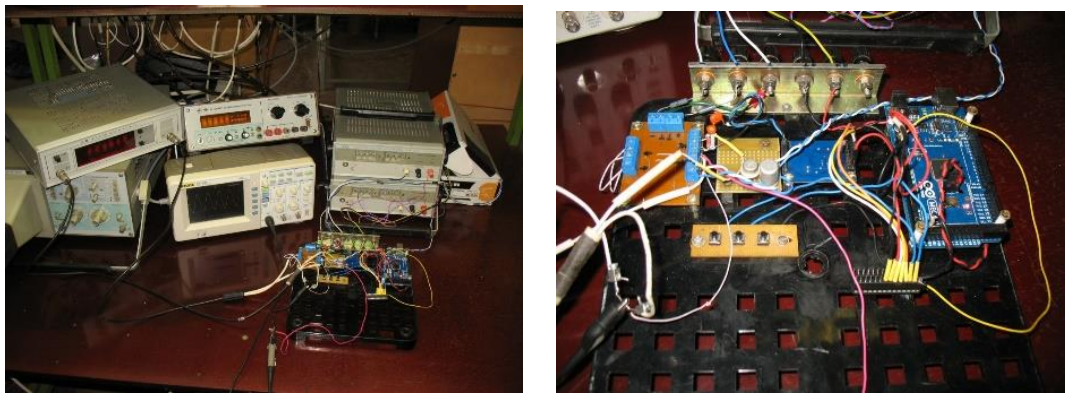

Fig.5. Laboratory equipment for the study of multifrequency phase method of measuring the distance of objects.

\section{Conclusions}

A s a result of the line study, the dependences of the amplitude and phase of the total reflected signal were obtained (Figs. 60 and 7). Fig.6,a and Fig.6,b show the dependences of the amplitude and phase of the total signal when the line is short-circuited at the end. Fig.7,a and Fig.7, b shows the dependences of the amplitude and phase of the total signal in the idle mode of the line 


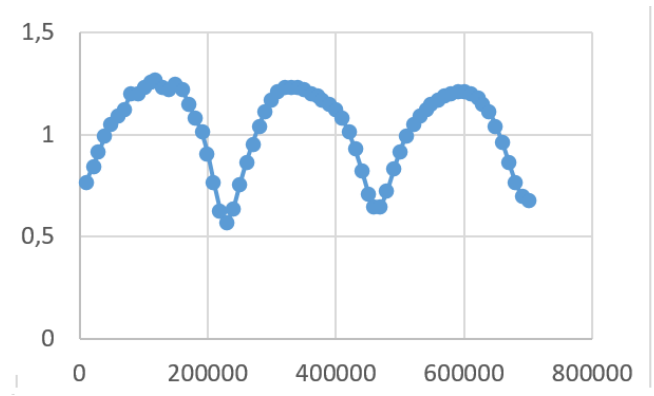

a)

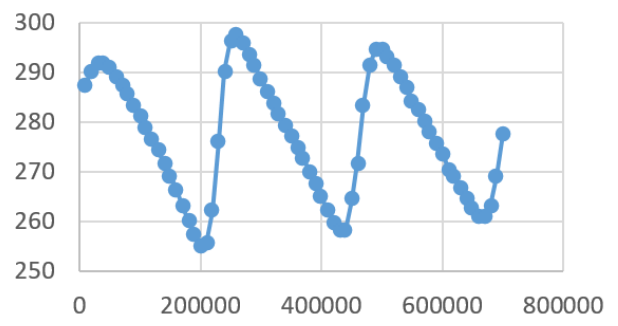

b)

Fig.6. D ependences of amplitude (a) and phase (b) on the frequency of the total signal in the mode of short circuit at the end of the line.

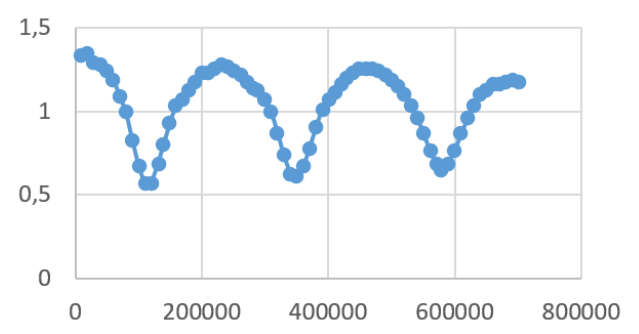

a)

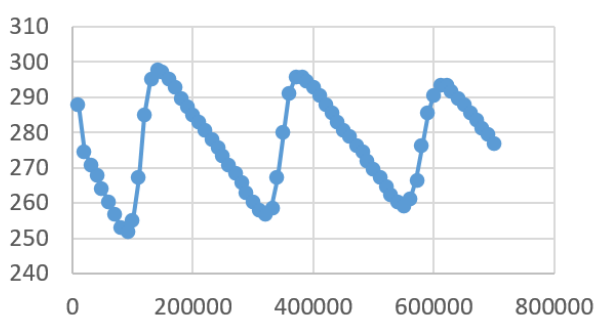

b)

Fig.7. Dependences of amplitude (a) and phase (b) on the frequency of the total signal in idle mode at the end of the line.

A nalysis of the passage of radio waves in subsurface environments with different electrophysical properties, allowed to develop a mathematical model for refining the results of measuring the distance of objects. The model consists in sequentially calculating the distance of objects using the velocity values calculated based on the electrical properties of the subsurface layer. The reflection coefficient at the interface of the two layers is to find the electrical properties of the subsurface layer. The obtained values are the dielectric constant of the layer material, the value of which is used to calculate the speed of propagation of the radio wave in the current layer.

A multiphase detection method has been proposed to generate diagnostic information that allows the reflected signals from several consecutive layers to be obtained. The reflection distance is determined for each layer. This system can be used to diagnose defects in multilayer structures that can transmit radio waves. A Iso it can be used for quality control of the put coverings and for the analysis of thickness of composite materials, isolation layers in the equipment.

\section{References}

1. Jyoti K. Sinha, Significance of Vibration Diagnosis of Rotating Machines during Installation and Commissioning: A Summary of Few Cases, Noise \& Vibration W orldwide, (2006)

2. V. Liubchyk, A. Kylimnik, S. Horyashchenko, Application of the Multi-frequency Phase Method of Ranging to Many Objects for Construction of Ground Penetrating Radar, International Radar Symposium (IRS-2013), D resden, Germany, 19 - 21 M ay, p. 835-840, (2013) 
3. V. Liubchyk, S. A. Karvan, G. B. Paraska, Model of transmission of probing signals in the study of nano-objects, M aterials Science 2012, 12th IEEE International Conference on Nanotechnology (IEEE-NAN O), ISSN : 1944-9399, (2012)

4. S. Horiashchenko, I. Golinka, A. Bubulis, V. Jurenas, Simulation and Research of the Nozzle with an UItrasonic Resonator for Spraying Polymeric M aterials, M echanika, Vol. 24, No 1, p. 61-64, (2018)

5. J. Musiał, S. Horiashchenko, R. Polasik, T. Kałaczyński, M. M atuszewski, M. Śrutek, A brasion W ear Resistance of Polymer Constructional M aterials for Rapid Prototyping and Tool-M aking Industry, Polymers, 12, p. 873, (2020)

6. S. Horiashchenko, K. Horiashchenko, J. Musiał, M ethodology of measuring spraying the droplet flow of polymers from nozzle, M echanika, 26, p. 82-86, (2020)

7. D. Abboud, Vibration-based condition monitoring of rotating machines in nonstationary regime, M echanics, IN SA de Lyon, (2015)

8. A. Boryssenko, V. Prokhorenko, Simulation of Subsurface Electromagnetic Wave Propagation and Scattering, Millenium Conference on A ntennas and Propagation, A pril 9-14, Davos, Switzerland, (2000)

9. Y u. K. Sirenko, I. V. Sukharevsky, O. I. Sukharevsky, N. P. Y ashina, Fundamental and applied problems of the theory of electromagnetic wave scattering, p. 344, K harkiv, (2000)

10. O. Paraska, S. K arvan, V. Liubchik, O. Chervonyuk, Nanoparticles in environment: life cycle and method of determination, N anosafety Congress - Turkey: A bstract book, 2628 A pril 2012, K emer-A ntalya, Turkey, p.56, (2012)

11. V. Liubchyk, S. Karvan, G. Paraska, M odel of Transmission of Probing Signals in the Study of Nano-objects, $12^{\text {th }}$ International Conference on Nanotechnology: Conference Proceedings, ICC, Birmingham, UK, 20-23, (2012)

12. K. Horiaschenko, V. Stetcuk, Modified method of spectral analysis of the reflection signal for damage detection systems in power lines, JV E International Ltd, (2018)

13. K. L. Horiaschenko, Pulse-phase measurements for a line with two inhomogeneities, $\mathrm{M}$ easuring and computing engineering in technological processes, $\mathrm{K}$ hmelnytskyi, $\mathrm{No}$. 1, p. 80-82, (2003)

14. Technical documantation, AD 9850. CMOS, 125 M Hz Complete DDS Synthesizer. A nalog Device, http://www.analog.com/media/en/technical-documentation/datasheets/A D 9850.pdf

15. W. Kester, M T-003: Understand SINAD, ENOB, SNR, THD, THD + N, and SFDR so You Don't Get Lost in the Noise Floor, Analog Devices, (2011)

16. M. Żółtowski, M. Liss, B. Żółtowski, J. Melcer, Truss harbor cranes modal design elements research, Polish M aritime Research, No 4 (88), V ol. 22, pp. 84-92 (2015)

17. M. Łukasiewicz, M. Liss, N. Dluhunovych, Analysis of vibrodiagnostics methods in the technical state study of designed multimedia mobile scenes, 18th International Conference Diagnostics of M achines and V ehicles, M ATEC W eb of Conferences, Vol. 302 (2019)

18. M. Łukasiewicz, P. Falęcki, T. Kałaczyński, B. Żółtowski, J. Musiał, J. Wilczarska, R. K ostek: A nalysis of the thermovision diagnostics potential in the light system elements, 24th International Conference Engineering M echanics, Engineering M echanics, 24, p. 1805-8248, Czech Republic, (2018)

19. V. Martynyuk, O. Eromenko, J. Boiko, T. Kałaczyński, Diagnostics of supercapacitors, 17th International Conference Diagnostics of $M$ achines and V ehicles, $M$ atec W eb of Conferences 2018, 182, 1-10, Poland, (2018)

20. M. Łukasiewicz, T. Kalaczynski, J. Musial, J. Shalapko, Diagnostics of buggy vehicle transmission gearbox technical state based on modal vibrations, JVE Inetrnational J ournal, V ol. 16, 6, p. 3137-3145, (2014) 